\title{
ROBUST AND EFFICIENT LARGE DEFORMATION ANALYSIS OF KIRCHHOFF-LOVE SHELLS: LOCKING, PATCH COUPLING AND ITERATIVE SOLUTION
}

\section{DOMENICO MAGISANO ${ }^{1}$, FRANCESCO S. LIGUORI ${ }^{1}$, LEONARDO LEONETTI ${ }^{1}$, ANTONIO MADEO ${ }^{1}$, GIOVANNI GARCEA ${ }^{1}$, JOSEF KIENDL ${ }^{2}$ AND ALESSANDRO REALI $^{3}$}

\author{
${ }^{1}$ Dipartimento di Ingegneria Informatica, Modellistica, Elettronica e Sistemistica \\ Università della Calabria, Italy, domenico.magisano@unical.it \\ ${ }^{2}$ Institute of Engineering Mechanics \& Structural Analysis Bundeswehr University Munich, Germany \\ ${ }^{3}$ Department of Civil Engineering and Architecture, University of Pavia, Italy
}

Key words: Large deformations, isogeometric analysis, Kirchhoff-Love shells, patch coupling, penalty, locking

\begin{abstract}
Isogeometric Kirchhoff-Love elements have received an increasing attention in geometrically nonlinear analysis of thin walled structures. They make it possible to meet the $C^{1}$ requirement in the interior of surface patches, to avoid the use of finite rotations and to reduce the number of unknowns compared to shear flexible models. Locking elimination, patch coupling and iterative solution are crucial points for a robust and efficient nonlinear analysis and represent the main focus of this work. Patch-wise reduced integrations are investigated to deal with locking in large deformation problems discretized via a standard displacement-based formulation. An optimal integration scheme for third order $C^{2}$ NURBS, in terms of accuracy and efficiency, is identified, allowing to avoid locking without resorting to a mixed formulation. The Newton method with mixed integration points (MIP) is used for the solution of the discrete nonlinear equations with a great reduction of the iterative burden and a superior robustness with respect to the standard Newton scheme. A simple penalty approach for coupling adjacent patches, applicable to either smooth or non-smooth interfaces, is proposed. An accurate coupling, also for a nonmatching discretization, is obtained using an interface-wise reduced integration while the MIP iterative scheme allows for a robust and efficient solution also with very high values of the penalty parameter.
\end{abstract}

\section{INTRODUCTION}

Isogeometric Kirchhoff-Love elements have received an increasing attention in geometrically nonlinear analysis of thin shells due to the high continuity of NURBS functions that makes it possible to meet the $C^{1}$ requirement in the interior of patches [1]. This kind of analysis spread rapidly in the scientific community. Among the main reasons for its success is the high order and continuity while practically maintaining the same number of degrees of freedom (DOFs) of linear Lagrangian interpolations and the exact geometric description of shells. These considerations make IGA very attractive, particularly in geometrically nonlinear analysis where a highly continuous solution is often expected, like for instance in buckling problems $[2,3]$. The standard displacement-based formulation is affected by locking when low order splines are used. Increasing the order of the shape functions reduces locking but, at the same 
time, increases the computational burden for the assembly of the discrete operators and for the solution of the discrete equations because of the decreasing stiffness matrix sparsity. Mixed formulations with continuous stress shape functions have been successfully proposed [4] to eliminate locking in linear and nonlinear problems, but the extra variables can be condensed only at the patch level and this is not convenient because leads to a fully populated stiffness matrix. An interesting alternative is the use of displacement formulations with patch-wise reduced integration rules [5, 6, 7]. In this case, locking is drastically reduced and its effects are made insignificant in most practical computations. Moreover, a significantly lower number of integration points is employed compared to standard Gauss quadrature, improving also the computational efficiency. In path-following methods for geometrically nonlinear analyses of beams and shells, many authors observed a more robust and efficient iterative solution for mixed formulations [8]. The performance of Newton's method drastically deteriorates in displacement formulations when the membrane/flexural stiffness ratios get higher [9, 10]. Mixed formulations are not affected by this drawback as the stress unknowns are used as independent variables in the iterative process. To eliminate this inconvenience in displacement-based formulations, the Mixed Integration Point (MIP) strategy has been recently proposed in [11]. It consists of a relaxation of the constitutive equations at each integration point during the Newton iterative process. Within a MIP framework, Newton's method can withstand much larger increments with a reduced number of iterations to obtain an equilibrium point compared to a standard Newton's strategy without the need of defining a stress interpolation [6, 12]. Engineering models of appreciable complexity are typically modeled using multiple surface patches and, often, neither rotational continuity nor conforming discretization can be practically obtained at patch interfaces. Despite the many potential advantages offered by isogeometric Kirchhoff-Love shells, they cannot be

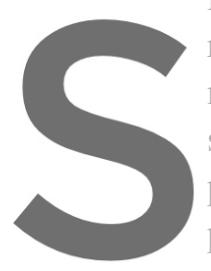
readily applied to such complex, multi-patch desig
nuity at patch interfaces. A simple penalty approad
smooth or non-smooth interfaces and either matchi
proposed in [13]. Both displacennent and totationa
penalty coefficient. This work seems very promis
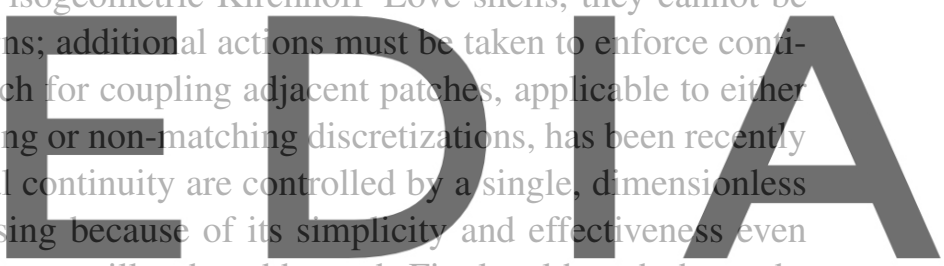

for complex geometries. However, two main issues are still to be addressed. Firstly, although the probRegister for freendence of the penalty coefficient can pe reduced by scaling factors which take inte account tween accuracy and numerical stability, which is typical for penalty formulations. This is the same issue experienced in finite rotation analysis of beams and shells, where the ill-conditioning is due to the significant difference between axial/membrane and flexural stiffness. In the penalty coupling approach, the ill-conditioning is due to the significant difference between coupling and patches stiffness. The second issue is that enforcing the constraints for a non-matching discretization could in general lead to an overconstrained interface and consequently to a wrong solution [14]. Locking elimination, patch coupling and iterative solution are crucial points for a robust and efficient nonlinear analysis of Kirchhoff-Love shells and represent the main focus of this work. Patch-wise reduced integrations are investigated to deal with locking in large deformation problems discretized via a standard displacement-based formulation. An optimal integration scheme for third order $C^{2}$ NURBS, in terms of accuracy and efficiency, is identified, allowing to avoid locking [15] without resorting to a mixed formulation. The Newton method with mixed integration points (MIP) is used for the solution of the discrete nonlinear equations with a great reduction in the iterative burden and a superior robustness with respect to the standard Newton scheme (large steps). A simple penalty approach for coupling adjacent patches, applicable to either smooth or non-smooth interfaces, is proposed. It is an improved version of the penalty approach in [13], able to 
avoid both the convergence difficulties in large deformation problems and the overconstraint issue for non-matching discretizations. An accurate coupling, also for the non-matching case, is obtained using an interface-wise reduced integration while the MIP iterative scheme allow for a robust and efficient solution also with very high values of the penalty parameter.

\section{THE ISOGEOMETRIC KIRCHHOFF-LOVE SHELL MODEL}

\subsection{Kirchhoff-Love shell kinematics}

We use a Total Lagrangian formulation to identify material points on the middle surface of the current configuration in terms of their position vector $\mathbf{X}(\xi, \eta)$ in the reference configuration and the displacement field $\mathbf{u}(\xi, \eta)$ :

$$
\mathbf{x}(\xi, \eta)=\mathbf{X}(\xi, \eta)+\mathbf{u}(\xi, \eta)
$$

where $\xi=[\xi, \eta]$ denotes convective curvilinear shell coordinates with $(\xi, \eta)$ representing in-plane coordinates. The middle surface covariant basis vectors in the undeformed and deformed configuration are obtained from the corresponding partial derivatives of the position vectors $\mathbf{X}$ and $\mathbf{x}$, respectively

$$
\mathbf{G}_{i}=\mathbf{X}_{, i}, \quad \mathbf{g}_{i}=\mathbf{x}_{, i}=\mathbf{G}_{i}+\mathbf{u}_{, i} \quad \text { with } i=1,2,
$$

where ()$, i$ denotes the partial derivative with respect to the $i$-th component of $\xi$, while the unit normal ones are

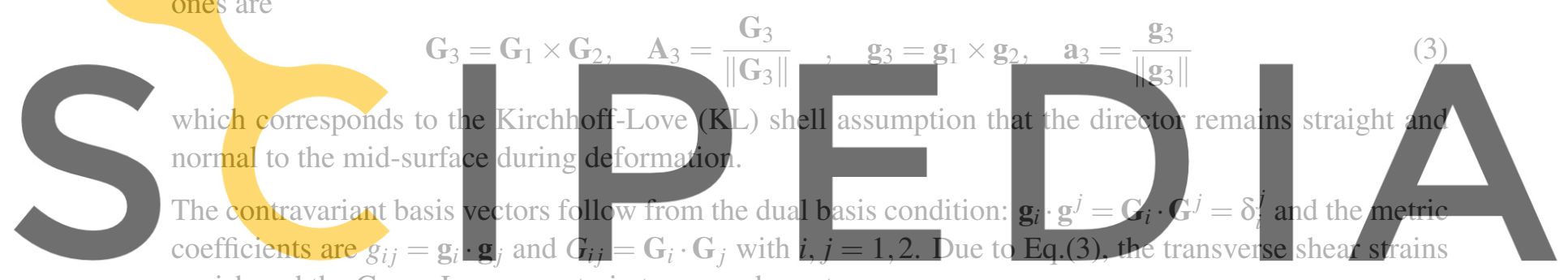

vanish and the Green-Lagrange strain tensor reduces to

\section{Register for free at https//www.scipedia.com to download the version without the watermark}

where $\bar{E}_{i j}$ are the covariant strain components. Assuming the strain to vary lineariy through the thickness, it is possible to split it into a constant membrane part and a linear bending one. The covariant strain coefficients are

$$
\bar{E}_{i j}=\bar{e}_{i j}+\zeta \bar{\chi}_{i j}=\frac{1}{2}\left(g_{i j}-G_{i j}\right)+\zeta\left(B_{i j}-b_{i j}\right) \quad \text { with } \quad i, j=1,2
$$

with $\zeta \in[-t / 2, t / 2]$ and $t$ the thickness of the shell. The curvature tensor coefficients are defined as in [1]

$$
\begin{aligned}
B_{i j} & =-\frac{1}{2}\left(\mathbf{G}_{i} \cdot \mathbf{A}_{3, j}+\mathbf{G}_{j} \cdot \mathbf{A}_{3, i}\right)=\mathbf{G}_{i, j} \cdot \mathbf{a}_{3}, \\
b_{i j} & =-\frac{1}{2}\left(\mathbf{g}_{i} \cdot \mathbf{a}_{3, j}+\mathbf{g}_{j} \cdot \mathbf{a}_{3, i}\right)=\mathbf{g}_{i, j} \cdot \mathbf{a}_{3} .
\end{aligned}
$$

A simplified third order strain measure providing the same results can be also adopted [15]. 


\subsection{The isogeometric shell element}

Following the isoparametric concept, geometry and displacement fields are approximated, over the element, as follows

$$
\mathbf{X}(\xi, \eta)=\mathbf{N}_{u}(\xi, \eta) \mathbf{X}_{e}, \quad \mathbf{u}(\xi, \eta)=\mathbf{N}_{u}(\xi, \eta) \mathbf{d}_{e}
$$

where $\mathbf{X}_{e}$ and $\mathbf{d}_{e}$ collect the element control points and the element control displacements, respectively. The matrix $\mathbf{N}_{u}(\xi, \eta)$ collects bivariate NURBS functions [16]. In this paper we deal only third order $C^{2}$ NURBS.

Adopting Voigt's notation, the covariant strain components in Eq.(5) are collected in the vector $\overline{\mathbf{E}}=$ $\left[\bar{E}_{\xi \xi}, \bar{E}_{\eta \eta}, 2 \bar{E}_{\xi \eta}\right]^{T}$, that, exploiting Eq.(6), becomes

$$
\overline{\mathbf{E}}=\overline{\mathbf{e}}+\zeta \bar{\chi}
$$

with $\overline{\mathbf{e}}=\left[\bar{e}_{\xi \xi}, \bar{e}_{\eta \eta}, 2 \bar{e}_{\xi \eta}\right]^{T}$ and $\overline{\boldsymbol{\chi}}=\left[\bar{\chi}_{\xi \xi}, \bar{\chi}_{\eta \eta}, 2 \bar{\chi}_{\xi \eta}\right]^{T}$.

The generalized stress components, once the kinematic model is assumed, are automatically given by assuring the invariance of the internal work. By collecting the contravariant stress components $\overline{\mathbf{S}} \equiv$ $\left[\bar{S}^{\xi} \xi, \bar{S}^{\eta \eta}, \bar{S}^{\xi} \eta\right]^{T}$, we can write

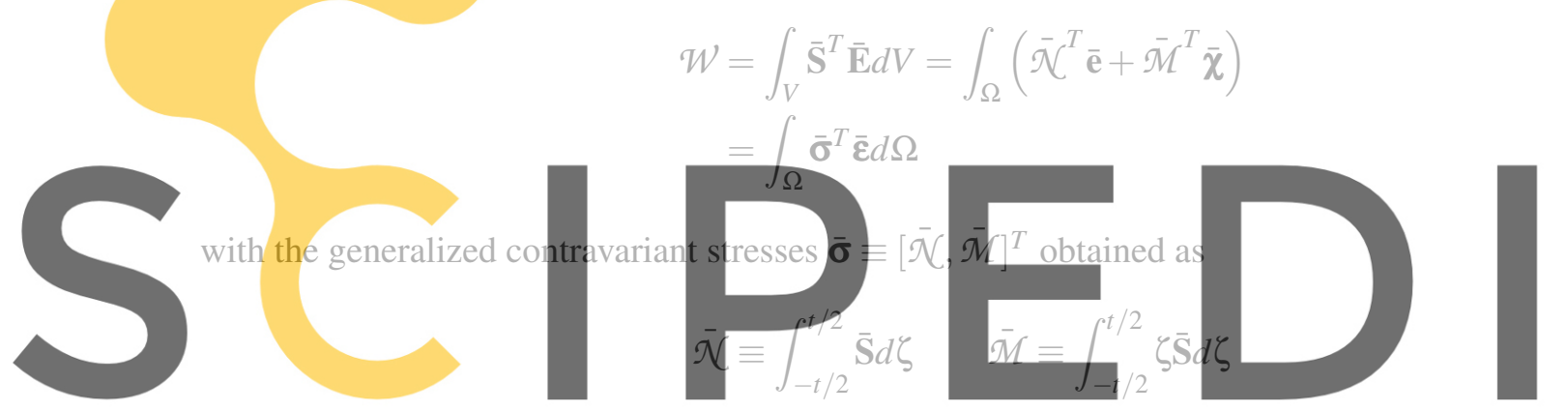

and the generalized covariant strain yector $\bar{\varepsilon} \equiv[\overline{\mathrm{e}}, \bar{\chi}]^{T}$. Exploiting the isogeometric approximation, $\bar{\varepsilon}$ Register fBeffree at https//www.scipedia.com to download the version without the watermark

$$
\bar{\varepsilon}=\overline{\mathbb{D}}\left(\xi, \eta, \mathrm{d}_{e}\right) \mathrm{d}_{e},
$$

where $\overline{\mathbf{D}}$ depends on the displacement DOFs.

For writing the constitutive equations with standard material matrices, we transform the generalized strains from the curvilinear coordinate system to a local Cartesian coordinate system whose $x-y$ plane is coincident with the mid-plane of the shell. For the Kirchhoff-Love shells we have furnishes the sought relationship:

$$
\begin{aligned}
\boldsymbol{\sigma} & =\mathbf{T}_{\sigma} \overline{\boldsymbol{\sigma}} \\
\boldsymbol{\varepsilon} & =\mathbf{T}_{\varepsilon} \overline{\boldsymbol{\varepsilon}}=\mathbf{T}_{\sigma}^{-T} \overline{\boldsymbol{\varepsilon}} \quad \text { with } \quad \mathbf{T}_{\sigma}=\left[\begin{array}{cc}
\mathbf{T}_{p} & 0 \\
0 & \mathbf{T}_{p}
\end{array}\right]
\end{aligned}
$$

where

$$
\mathbf{T}_{p}=\left[\begin{array}{ccc}
x_{\xi}^{2} & x_{\eta}^{2} & 2 x_{\xi} x_{\eta} \\
y_{\xi}^{2} & y_{\eta}^{2} & 2 y_{\xi} y_{\eta} \\
x_{\xi} y_{\xi} & x_{\eta} y_{\eta} & x_{\xi} y_{\eta}+x_{\eta} y_{\xi}
\end{array}\right]
$$


with $x_{\xi}=\mathbf{i}_{1}^{T} \mathbf{G}_{1}, y_{\xi}=\mathbf{i}_{2}^{T} \mathbf{G}_{1}, x_{\eta}=\mathbf{i}_{1}^{T} \mathbf{G}_{2}, y_{\eta}=\mathbf{i}_{1}^{T} \mathbf{G}_{2} ; \mathbf{i}_{1}$ and $\mathbf{i}_{2}$ are the unit vectors along the axis of the local Cartesian coordinates where the material properties are assigned.

Eq.(7) and (10) in Cartesian components become respectively

$$
\mathbf{E}=\mathbf{e}+\zeta \chi \quad \text { and } \quad \boldsymbol{\varepsilon}=\mathbf{D}\left(\xi, \eta, \mathbf{d}_{e}\right) \mathbf{d}_{e} .
$$

The homogenized material law of the shell can be expressed as

$$
\begin{gathered}
\boldsymbol{\sigma}=\mathbf{C}_{\varepsilon} \boldsymbol{\varepsilon} \quad \mathbf{C}_{\varepsilon}=\left[\begin{array}{ll}
\mathbf{C}_{e e} & \mathbf{C}_{e \chi} \\
\mathbf{C}_{e \chi}^{T} & \mathbf{C}_{\chi \chi}
\end{array}\right] \\
\mathbf{C}_{e e}=\sum_{k} t_{k} \mathbf{C}_{k}, \quad \mathbf{C}_{e \chi}=\sum_{k} z_{k} t_{k} \mathbf{C}_{k}, \quad \mathbf{C}_{\chi \chi}=\sum_{k}\left(\frac{t_{k}^{3}}{12}+t_{k} z_{k}^{2}\right) \mathbf{C}_{k}
\end{gathered}
$$

where the sum is on the number of layers, $t_{k}$ is the thickness of the $k$-th ply, $z_{k}$ is the distance between the centroid of the $k$-th ply and the mid-plane of the laminate and $\mathbf{C}_{k}$ is the lamina constitutive matrix.

\subsection{Nonlinear analysis}

In displacement-based formulations, the strain energy can be expressed as a sum of element contributions $\Phi(\mathbf{d}) \equiv \sum_{e} \Phi_{e}\left(\mathbf{d}_{e}\right)$
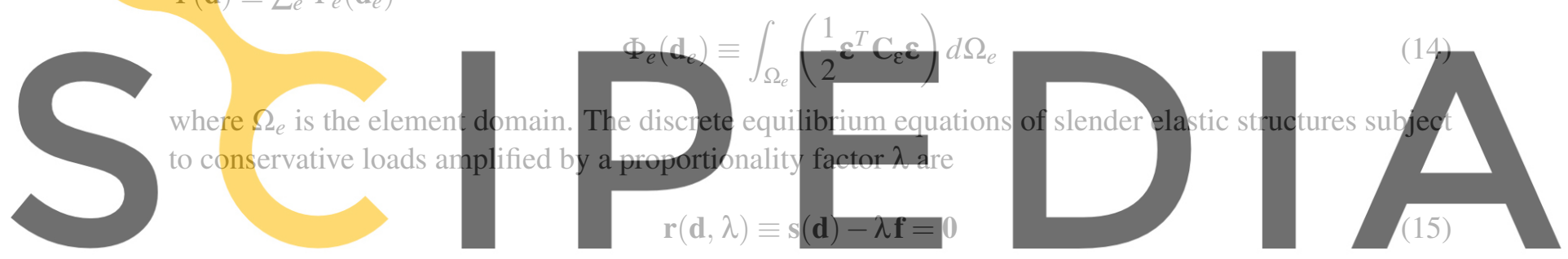

where $\mathbb{r}: \mathbb{R}^{N+1} \rightarrow \mathbb{R}^{N}$ is a nonlinear vectorial function of the vector $\mathbf{z} \equiv\left\{\mathbf{d}_{\mathbf{}} \lambda\right\} \in \mathbb{R}^{N+1}$, collecting the

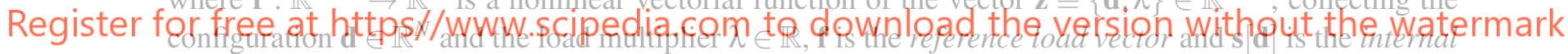

force vector, i.e. the strain energy gradient with respect to d. Eq.(15) represents a system of $N$ equations and $N+1$ unknowns and its solutions define the equilibrium paths as curves in $\mathbb{R}^{N+1}$. The Riks' approach [17] can be used to trace these curves step-by-step from a known initial configuration $\mathbf{d}_{0}$ corresponding to $\lambda=0$. At each step some Newton iterations are needed to solve (15). To this end we also define the tangent stiffness matrix as the strain energy Hessian with respect to d, which at element level assumes the following form

$$
\mathbf{K}_{e}\left(\mathbf{d}_{e}\right) \equiv \int_{\Omega_{e}}\left(\mathbf{B}\left(\mathbf{d}_{e}\right)^{T} \mathbf{C}_{\varepsilon} \mathbf{B}\left(\mathbf{d}_{e}\right)+\Gamma\left(\mathbf{d}_{e}, \boldsymbol{\sigma}\left(\mathbf{d}_{e}\right)\right)\right) d \Omega_{e} .
$$

In order to avoid locking, the patch-wise reduced integrations proposed in [15] are used to evaluate the integrals.

\subsection{The iterative scheme with mixed integration points}

In $[10,9]$, it is shown that the Newton's method convergence for displacement-based formulations gets slower and requires a smaller step size when the slenderness of the structure increases. This fact is 
unrelated to the accuracy of the interpolation and occurs because the stresses $\boldsymbol{\sigma}_{g}\left(\mathbf{d}_{e}\right)$, used to evaluate the tangent stiffness matrix $\mathbf{K}_{e}\left(\boldsymbol{\sigma}_{g}\left(\mathbf{d}_{e}\right), \mathbf{d}_{e}\right)$, are forced to satisfy the constitutive equations at each iteration. In [11], a strategy called Mixed Integration Point has been proposed in order to overcome these limitations in standard displacement-based finite element problems and then extended and tested in displacement-based isogeometric formulations [6,12]. The fundamental idea of the MIP Newton scheme is to relax the constitutive equations at the level of each integration point during the iterations. This is made by rewriting the strain energy in a pseudo Hellinger-Reissner form on the element:

$$
\Phi_{e}\left(\mathbf{d}_{e}\right) \equiv \sum_{g=1}^{n}\left(\boldsymbol{\sigma}_{g}^{T} \boldsymbol{\varepsilon}_{g}\left(\mathbf{d}_{e}\right)-\frac{1}{2} \boldsymbol{\sigma}_{g}^{T} \mathbf{C}_{g}^{-1} \boldsymbol{\sigma}_{g}\right) w_{g}
$$

where the stresses at each integration point $\boldsymbol{\sigma}_{g}$ are now independent variables, which however can be condensed out at the element level without any additional cost. Details on the method formulation are reported in $[11,6]$. The MIP iterative scheme is very close to the standard Newton's one for purely displacement-based models. The main difference consists of the different values of the stresses at the integration points used for the evaluation of the tangent stiffness matrix. These stresses are independent variables which are directly predicted as extrapolation of the previous equilibrium points, just as the displacements, and updated during the iterations with the correction term

$$
\dot{\boldsymbol{\sigma}}_{g}=\mathbf{C}_{g} \mathbf{B}_{g}^{j} \dot{\mathbf{d}}_{e}+\mathbf{C}_{g} \boldsymbol{\varepsilon}_{g}^{j}-\boldsymbol{\sigma}_{g}^{j}
$$

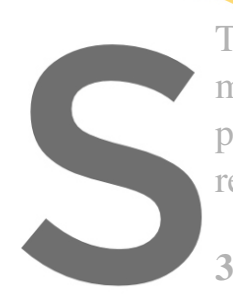

The stresses are defined

means that the computation proved that the replacem

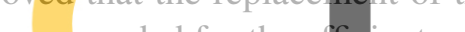

\section{PENALTY COUPLING OH}
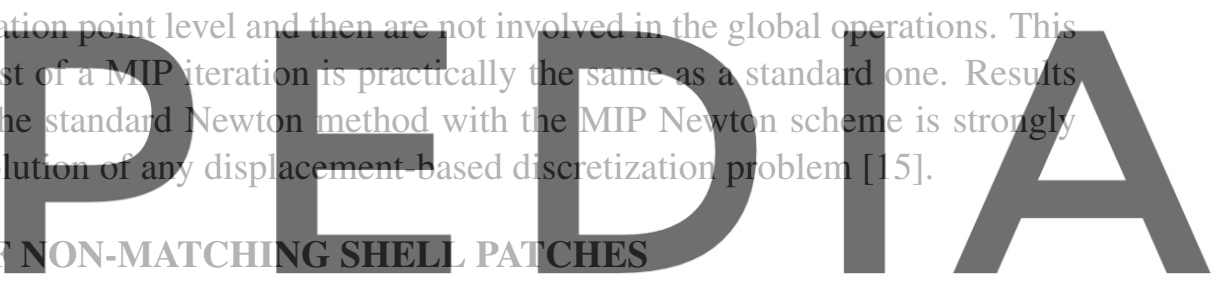

In the following, after recalling briefly the penalty approach in [13] to couple multiple non-matching

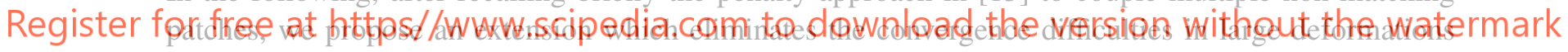
and improve the accuracy for non-matching cases.

\subsection{Displacement-based penalty formulation}

In the following, it is assumed that there are two patches, $A$ and $B$, with two edges which, in the undeformed configuration, are approximately co-located along an interface curve $\ell$. For enforcing displacement continuity between the two patches, the following penalty energy [13] is added to the total energy:

$$
W_{d}=\frac{1}{2} \int_{\ell} \alpha_{d}\left(\mathbf{u}^{A}-\mathbf{u}^{B}\right)^{T}\left(\mathbf{u}^{A}-\mathbf{u}^{B}\right)
$$

where superscripts $\mathrm{A}$ and $\mathrm{B}$ indicate quantities evaluated on the common edge of patches $A$ or $B$ respectively, $\alpha_{d}$ is a penalty parameter, further discussed in the following, large enough to dictate that, if the distance between points belonging to the common edge of $A$ and $B$ is not the same in the deformed and undeformed configurations, a large penalty energy is introduced into the system. The coupling methodology must also maintain the angle formed by patches $A$ and $B$. Analogously, for imposing rotational continuity between the two patches, using the unit vectors defined in Fig.1, the following penalty energy 


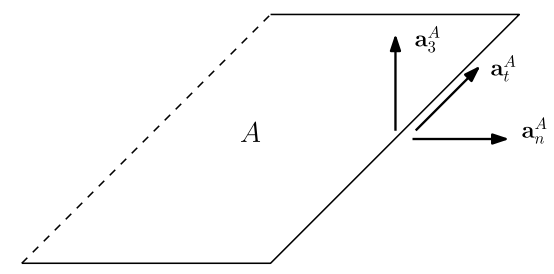

Figure 1: Illustration of vectors $\mathbf{a}_{3}$ and $\mathbf{a}_{n}$.

is further introduced:

$$
\begin{aligned}
W_{r}=\frac{1}{2} \int_{\ell} \alpha_{\ell} & \left(\left(\mathbf{a}_{3}^{A} \cdot \mathbf{a}_{3}^{B}-\mathbf{A}_{3}^{A} \cdot \mathbf{A}_{3}^{B}\right)\left(\mathbf{a}_{3}^{A} \cdot \mathbf{a}_{3}^{B}-\mathbf{A}_{3}^{A} \cdot \mathbf{A}_{3}^{B}\right)\right. \\
& \left.+\left(\mathbf{a}_{n}^{A} \cdot \mathbf{a}_{3}^{B}-\mathbf{A}_{n}^{A} \cdot \mathbf{A}_{3}^{B}\right)\left(\mathbf{a}_{n}^{A} \cdot \mathbf{a}_{3}^{B}-\mathbf{A}_{n}^{A} \cdot \mathbf{A}_{3}^{B}\right)\right)
\end{aligned}
$$

where $\alpha_{r}$ is a large enough penalty parameter. The extension to multiple patches is trivial and requires only the addition of the corresponding coupling energies. A key drawback of penalty methods is that the penalty parameters are problem dependent, must be high enough to ensure constraint satisfaction but not too high to create excessive ill-conditioning. The selection of penalty parameters, usually performed empirically by the analyst, has a strong influence on the solution quality. Problem dependence can be reduced taking into account geometry and material properties in the selection. The displacement and

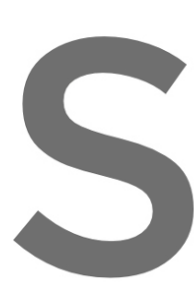
rotation penalty parameters

stiffnesses, respectively stiffness properties:

where $\alpha$ is a single pen
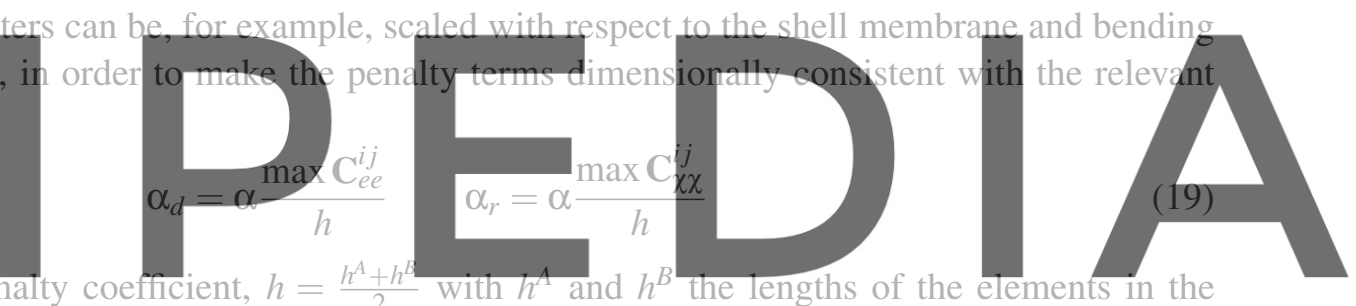

direction most parallel to the coupling curve and $i, j=1,2$. With the scaling terms (19), the penalty term

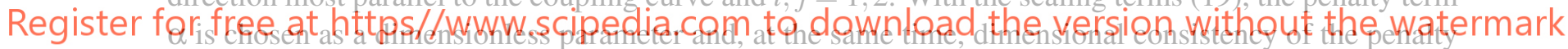
energy is guaranteed [13].

\subsection{Penalty formulation with reduced integration and mixed integration points}

An exact integration of the penalty energy for the displacement penalty approach can cause overconstrained interfaces with consequent inaccurate results. As the overconstrained coupling can be interpreted as a form of locking, we propose the use of an interface-wise reduced integration for the displacementbased approach. After an intense experimental campaign we selected the following rule: integration points and weights to be used are those which exactly integrate a target space $S_{c}^{q}$ along the interface of the patch with coarsest mesh of order $q=2 p-1$ and regularity $c=p-1$, with $p$ the order of the isogeometric approximation functions on such a patch. Integration points and weights are easily provided by the algorithm given in [5]. The proposed reduced integration rule is accurate and does not give stability problems [18]. However, this does not solve the convergence issue in large deformation problems for high values of the penalty parameter. In order to avoid the convergence difficulties without explicitly introducing the approximation of the Lagrange multipliers work-conjugated to the coupling equations, since the computation of the penalty energy is performed by numerical integration, we can exploit the 
Table 1: Wingbox: stacking sequence.

\begin{tabular}{cccc}
\hline Skin bay & Skin & Stiffener & Spar web \\
\hline $90 /[(0 \pm\langle 52 \mid 35\rangle) / 0 / \pm 45]_{S}$ & $90 /[ \pm 35 / 0 / \pm 45]_{S}$ & {$\left[90 / 45 / 0_{2} /-45 / 0\right]_{S}$} & $90 /\left[ \pm 35 / 0_{3} / \pm 45\right]_{S}$ \\
\hline
\end{tabular}

mixed integration point strategy

$$
\begin{gathered}
W_{d}=\sum_{e} W_{d e} \quad \text { with } \quad W_{d e}=\sum_{g}\left(\mathbf{q}_{g}^{T} \mathbf{v}_{g}-\frac{1}{2} \mathbf{q}_{g}^{T} \mathbf{h}_{d g} \mathbf{q}_{g}\right) w_{g} \\
W_{r}=\sum_{e} W_{r e} \quad \text { with } \quad W_{r e}=\sum_{g}\left(\mathbf{r}_{g}^{T} \mathbf{w}_{g}-\frac{1}{2} \mathbf{r}_{g}^{T} \mathbf{h}_{r g} \mathbf{r}_{g}\right) w_{g}
\end{gathered}
$$

where the values of the Lagrangian fields at the integration points $\mathbf{q}_{g}$ and $\mathbf{r}_{g}$ are used as independent variables in the iterative solution, directly predicted and corrected. In this case, by adding $W_{d}$ and $W_{r}$, evaluated as in Eq.(20) and Eq.(21), to the total energy of the structure, we can now directly apply the MIP Newton iteration method described in the previous section to solve the global discrete equations. $\mathbf{q}_{g}$ and $\mathbf{r}_{g}$ are condensed out as for the mixed penalty formulation after linearization of the stationarity condition. However, the condensation is now local at each integration point exploiting the local definition of $\mathbf{q}_{g}$ and $\mathbf{r}_{g}$. This means that, as already explained in the previous section, they are not involved in the definition of the global residual vector, which remains the same as in the classical displacement

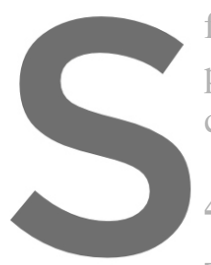
formulation [13]. However. performance of the iterativ can now use high value

4 NUMERICAL TEST: A VARIABLE ANGLE T

The numerical example regards a full-scale composite wingbox, that has been recently designed, man-

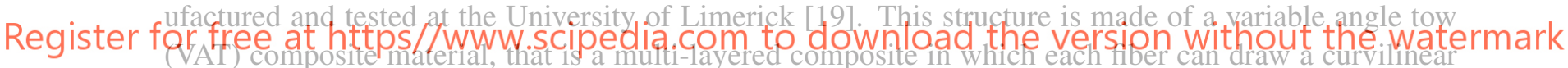
path $[19,20]$. Hereafter, the wingbox is analyzed using the proposed multi-patch IGA Kirchhoff-Love model named RMIP. We show how, thanks to the multi-patch coupling algorithm [18], the KirchhoffLove model can be successfully employed on structures composed by many panels and the solution accuracy within each patch due to the patch-wise reduced integration [15]. Additionally, it is shown that the use of a non-matching discretization can further reduce the DOFs of the numerical problem, thereby speeding-up the geometrically nonlinear analysis of a full-scale structure. The material properties of the ply, having thickness of $0.1875 \mathrm{~mm}$, are $E_{1}=135.00 \mathrm{GPa}, E_{2}=7.54 \mathrm{GPa}, G_{12}=5.00 \mathrm{GPa}$ and $v_{12}=0.30$. The fiber orientations are measured, according to the local reference systems reported in Fig.2, from the $e_{1}$ axis, in the direction of $e_{3}$. The VAT laminate is characterized by linear variation of the fiber orientations. According to the notation suggested by Gürdal and Olmedo [21], Tab.1 shows the stacking sequence of the main parts of the wingbox. The skin is composed by 11 layers, while 4 additional layers have been added to the spar web to improve the resistance against shear-buckling. It is possible to note from Fig.3 how the thickness change is realized on the skin near the corner and a zone composed by 13 layers has been added to make it smoother. More details can be found in [19]. The wingbox is considered to be loaded on one end by a shear force $F_{A}=23.8 \mathrm{kN}$ and a flexural moment 


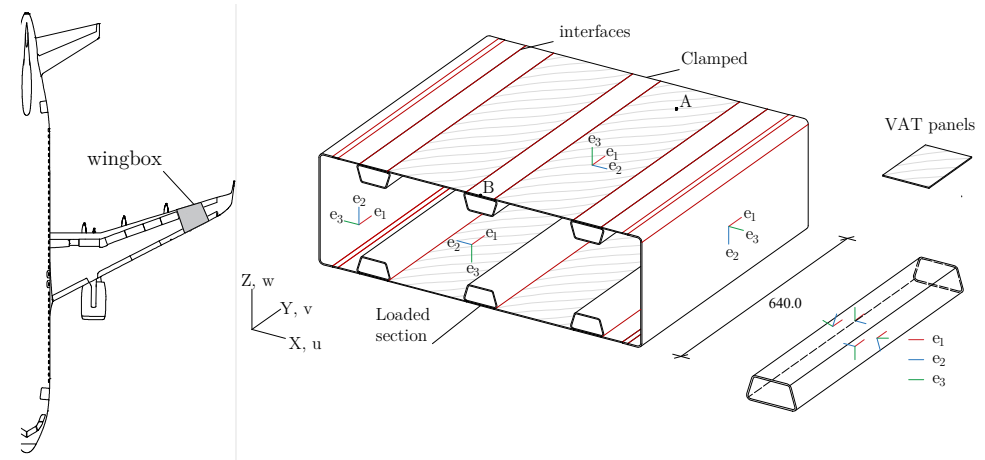

Figure 2: Wingbox: geometry and boundary conditions. The interface lines are marked in red.

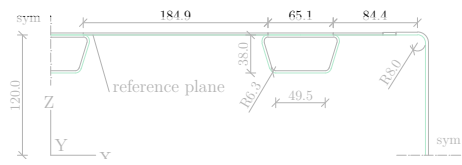

Figure 3: Wingbox: detailed view of the cross-section. A green line defines the shell reference planes.

$M_{A}=14.28 \mathrm{kNm}$, while the opposite side is fully clamped. The loaded section is modeled to behave

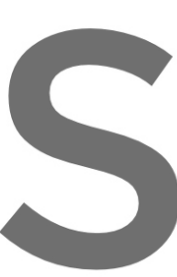
rigidly. The stiffnes

mid-plane of the panels no need for rigid links

buckling waves on the

on the skin bay to obtai

two meshes are adopted
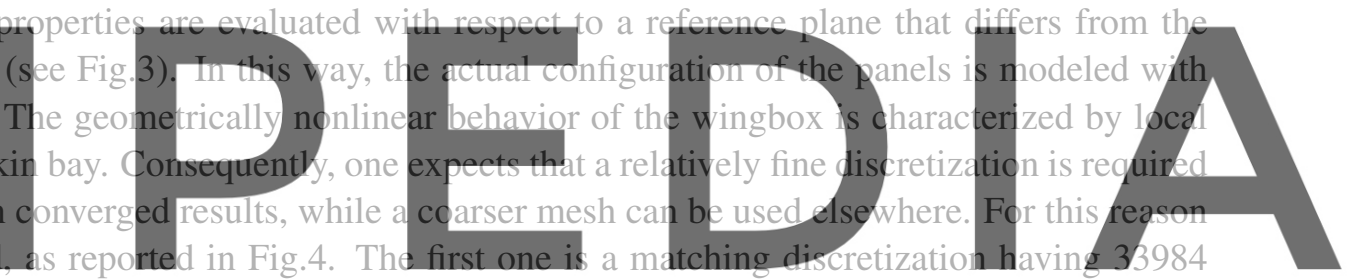

DOFs. Starting from the first mesh, by reducing the number of subdivisions of stiffeners, skin, and spar

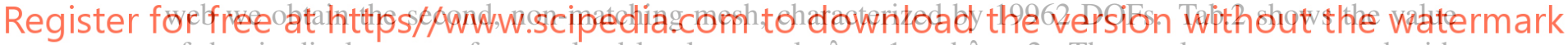
of the tip displacement for two load levels, namely $\lambda=1$ and $\lambda=2$. The results are compared with the solution provided by a very fine mesh of S4R elements from ABAQUS and that obtained using a $C^{0}$

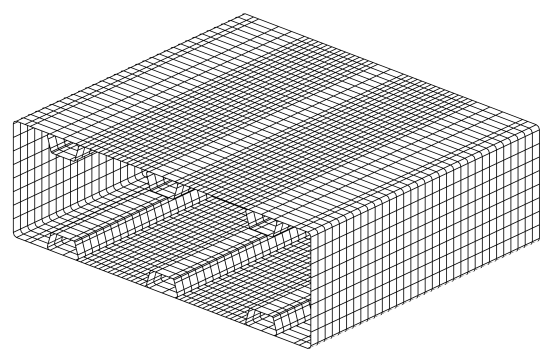

Matching (33984 DOFs)

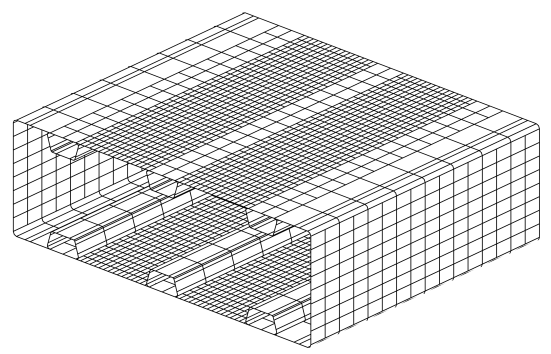

Non-matching (19962 DOFs)

Figure 4: Wingbox: matching discretization and non-matching discretization. 
Table 2: Wingbox: comparison of the tip displacement at two different load levels for different models.

\begin{tabular}{cccc}
\hline model & $\alpha$ & $w_{B}(\lambda=1)$ & $w_{B}(\lambda=2)$ \\
\hline RMIP, mesh (a) & $10^{3}$ & 2.131 & 4.339 \\
RMIP, mesh (b) & $10^{3}$ & 2.182 & 4.443 \\
RMIP, mesh (a) & $10^{6}$ & 1.661 & 3.374 \\
RMIP, mesh (b) & $10^{6}$ & 1.649 & 3.350 \\
ABAQUS S4R & - & 1.664 & 3.403 \\
solid-shell FE [19] & - & 1.657 & 3.389 \\
\hline
\end{tabular}

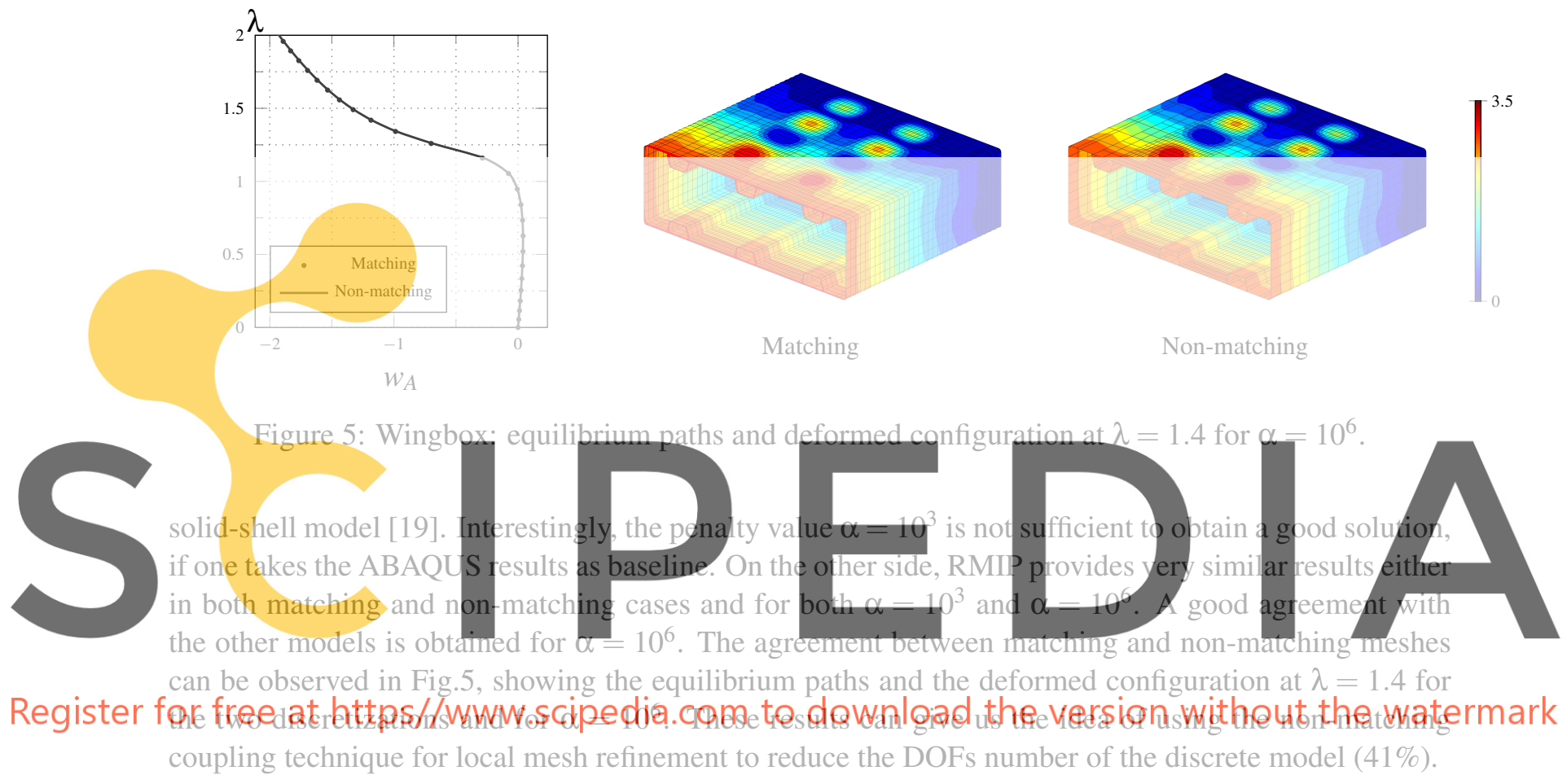

\section{Conclusions}

This work showed how to perform an accurate, efficient and robust analysis of thin-walled structures in large deformations modeled as assemblage of Kirchhoff-Love shell patches. An isogeometric discretization with third order $C^{2}$ NURBS is employed to describe geometry and displacement field within each patch. An accurate patch-wise reduced integration was found to avoid locking and reduce significantly the total number of integration points. A simple penalty method was given to impose displacement and rotational continuity in multi-patch structures. An interface-wise reduced integration provides an accurate coupling also for non-matching meshes. Finally, the Newton method with mixed integration points gives robustness and efficiency in the iterative solution. Large load steps can be used in path-following analyses with a reduced number of iterations to achieve equilibrium, also for very slender structures and high penalty coefficients. More details can be found in $[15,18]$. 


\section{REFERENCES}

[1] J. Kiendl, K.-U. Bletzinger, J. Linhard, R. Wüchner, Isogeometric shell analysis with kirchhoff-love elements, Computer Methods in Applied Mechanics and Engineering 198 (49) (2009) 3902 - 3914. doi:https://doi.org/10.1016/j.cma.2009.08.013.

[2] G. Garcea, L. Leonetti, D. Magisano, R. Gonçalves, D. Camotim, Deformation modes for the postcritical analysis of thin-walled compressed members by a Koiter semi-analytic approach, International Journal of Solids and Structures 110-111 (2017) 367-384. doi:10.1016/j.ijsolstr.2016.09.010.

[3] G. Garcea, F. S. Liguori, L. Leonetti, D. Magisano, A. Madeo, Accurate and efficient a posteriori account of geometrical imperfections in Koiter finite element analysis, International Journal for Numerical Methods in Engineering 112 (9) (2017) 1154-1174, nme.5550. doi:10.1002/nme.5550.

[4] R. Echter, B. Oesterle, M. Bischoff, A hierarchic family of isogeometric shell finite elements, Computer Methods in Applied Mechanics and Engineering 254 (2013) 170-180. doi:10.1016/j.cma.2012.10.018.

[5] K. A. Johannessen, Optimal quadrature for univariate and tensor product splines, Computer Methods in Applied Mechanics and Engineering 316 (2017) 84 - 99, special Issue on Isogeometric Analysis: Progress and Challenges. doi:http://doi.org/10.1016/j.cma.2016.04.030.

[6] L. Leonetti, F. Liguori, D. Magisano, G. Garcea, An efficient isogeometric solid-shell formulation for geometrically nonlinear analysis of elastic shells, Computer Methods in Applied Mechanics and Engineering 331 (2018) 159 - 183. doi:https://doi.org/10.1016/j.cma.2017.11.025.

[7] L. Leonetti, D. Magisano, F. Liguori, G. Garcea, An isogeometric formulation of the koiter's theory for buckling and initial post-buckling analysis of composite shells, Computer Methods in Applied Mechanics and Engineering 337 (2018) 387 - 410. doi:https://doi.org/10.1016/j.cma.2018.03.037.

[8] D. Magisano, K. Liang, G. Garcea, L. Leonetti, M. Ruess, An efficient mixed variational reducedorder model formulation for nonlinear analyses of elastic shells, International Journal for Numerical Methods in Engineering 113 (4) (2018) 634-655. doi:https://doi.org/10.1002/nme.5629.

[9] D. Magisano, L. Leonetti, G. Garcea, Advantages of the mixed format in geometrically nonlinear analysis of beams and shells using solid finite elements, International Journal for Numerical Methods in Engineering 109 (9) (2017) 1237-1262. doi:10.1002/nme.5322.

[10] G. Garcea, G. Trunfio, R. Casciaro, Mixed formulation and locking in path-following nonlinear analysis, Computer Methods in Applied Mechanics and Engineering 165 (1-4) (1998) 247-272.

[11] D. Magisano, L. Leonetti, G. Garcea, How to improve efficiency and robustness of the Newton method in geometrically non-linear structural problem discretized via displacement-based finite elements, Computer Methods in Applied Mechanics and Engineering 313 (2017) 986 - 1005. doi:http://dx.doi.org/10.1016/j.cma.2016.10.023.

[12] F. Maurin, F. Greco, S. Dedoncker, W. Desmet, Isogeometric analysis for nonlinear planar kirchhoff rods: Weighted residual formulation and collocation of the strong form, Computer Methods in Applied Mechanics and Engineering 340 (2018) 1023 - 1043. doi:https://doi.org/10.1016/j.cma.2018.05.025.

[13] A. J. Herrema, E. L. Johnson, D. Proserpio, M. C. Wu, J. Kiendl, M.-C. Hsu, Penalty coupling 
of non-matching isogeometric kirchhoff-love shell patches with application to composite wind turbine blades, Computer Methods in Applied Mechanics and Engineering 346 (2019) 810 - 840. doi:https://doi.org/10.1016/j.cma.2018.08.038.

[14] L. Coox, F. Maurin, F. Greco, E. Deckers, D. Vandepitte, W. Desmet, A flexible approach for coupling nurbs patches in rotationless isogeometric analysis of kirchhoff-love shells, Computer Methods in Applied Mechanics and Engineering 325 (2017) 505 - 531. doi:https://doi.org/10.1016/j.cma.2017.07.022.

[15] L. Leonetti, D. Magisano, A. Madeo, G. Garcea, J. Kiendl, A. Reali, A simplified kirchhoff-love large deformation model for elastic shells and its effective isogeometric formulation, Computer Methods in Applied Mechanics and Engineering 354 (2019) 369 - 396. doi:https://doi.org/10.1016/j.cma.2019.05.025.

[16] J. A. Cottrell, T. J. R. Hughes, Y. Bazilevs, Isogeometric Analysis: Toward Integration of CAD and FEA, 2009. doi:978-0-470-74873-2.

[17] E. Riks, An incremental approach to the solution of snapping and buckling problems, International Journal of Solids and Structures 15 (7) (1979) 529-551. doi:10.1016/0020-7683(79)90081-7.

[18] L. Leonetti, F. S. Liguori, D. Magisano, J. Kiendl, A. Reali, G. Garcea, A robust penalty coupling of non-matching isogeometric kirchhoff-love shell patches in large deformations, Computer Methods in Applied Mechanics and Engineering 371 (2020) 113289. doi:https://doi.org/10.1016/j.cma.2020.113289.

[19] F. S. Liguori, G. Zucco, A. Madeo, D. Magisano, L. Leonetti, G. Garcea, P. M. Weaver, Postbuckling optimisation of a variable angle tow composite wingbox using a multi-modal Koiter approach, Thin-Walled Structures 138 (2019) 183-198. doi:https://doi.org/10.1016/j.tws.2019.01.035.

[20] F. S. Liguori, A. Madeo, D. Magisano, L. Leonetti, G. Garcea, Post-buckling optimisation strategy of imperfection sensitive composite shells using Koiter method and Monte Carlo simulation, Composite Structures 192 (2018) 654 - 670. doi:https://doi.org/10.1016/j.compstruct.2018.03.023.

[21] Z. Gürdal, R. Olmedo, In-plane response of laminates with spatially varying fiber orientations - variable stiffness concept, AIAA Journal 31 (4) (1993) 751-758. doi:https://doi.org/10.2514/3.11613. 